\title{
Volume Bragg grating external cavities for the passive phase locking of high- brightness diode laser arrays: theoretical and experimental study
}

Paboeuf, David; Vijayakumar, Deepak; Jensen, Ole Bjarlin; Thestrup Nielsen, Birgitte; Lim, Jun; Sujecki, Slawomir; Larkins, Eric; Lucas-Leclin, Gaelle; Georges, Patrick

Published in:

Optical Society of America. Journal B: Optical Physics

Link to article, DOI:

10.1364/JOSAB.28.001289

Publication date:

2011

Document Version

Publisher's PDF, also known as Version of record

Link back to DTU Orbit

Citation (APA):

Paboeuf, D., Vijayakumar, D., Jensen, O. B., Thestrup Nielsen, B., Lim, J., Sujecki, S., Larkins, E., Lucas-Leclin, G., \& Georges, P. (2011). Volume Bragg grating external cavities for the passive phase locking of high-

brightness diode laser arrays: theoretical and experimental study. Optical Society of America. Journal B: Optical Physics, 28(5), 1289-1299. https://doi.org/10.1364/JOSAB.28.001289

\section{General rights}

Copyright and moral rights for the publications made accessible in the public portal are retained by the authors and/or other copyright owners and it is a condition of accessing publications that users recognise and abide by the legal requirements associated with these rights.

- Users may download and print one copy of any publication from the public portal for the purpose of private study or research.

- You may not further distribute the material or use it for any profit-making activity or commercial gain

- You may freely distribute the URL identifying the publication in the public portal 


\title{
Volume Bragg grating external cavities for the passive phase locking of high-brightness diode laser arrays: theoretical and experimental study
}

\author{
David Pabœuf, ${ }^{1}$ Deepak Vijayakumar, ${ }^{2}$ Ole Bjarlin Jensen, ${ }^{2}$ Birgitte Thestrup, ${ }^{2}$ Jun Lim, ${ }^{3}$ \\ Slawomir Sujecki, ${ }^{3}$ Eric Larkins, ${ }^{3}$ Gaëlle Lucas-Leclin,, ${ }^{1, *}$ and Patrick Georges ${ }^{1}$ \\ ${ }^{1}$ Laboratoire Charles Fabry de l'Institut d'Optique, CNRS, Univ Paris-Sud, \\ Campus Polytechnique-RD128-91127 Palaiseau Cedex, France \\ ${ }^{2}$ Technical University of Denmark, Department of Photonics Engineering, Frederiksborgvej 399, 4000 Roskilde, Denmark \\ ${ }^{3}$ Electrical Systems and Optics Research Division, University of Nottingham, University Park, Nottingham NG7 2RD, UK \\ ${ }^{*}$ Corresponding author: gaelle.lucas-leclin@institutoptique.fr
}

Received October 18, 2010; revised March 1, 2011; accepted March 2, 2011; posted March 15, 2011 (Doc. ID 136687); published April 27, 2011

\begin{abstract}
We describe the theoretical modeling of the external-cavity operation of a phase-locked array of diode lasers in two configurations, the self-imaging cavity based on the Talbot effect and the angular-filtering cavity. Complex filtering functions, such as the transmission or reflection of a volume Bragg grating (VBG), may be introduced in the external-cavity description. Experiments with high-brightness diode laser arrays were also conducted. The experimental results are carefully analyzed with regard to the numerical simulations, and the beneficial effect of the spectral selectivity of VBGs is demonstrated. @ 2011 Optical Society of America

OCIS codes: $140.2010,140.3298$
\end{abstract}

\section{INTRODUCTION}

Their outstanding electrical-optical efficiency and their unbeatable compactness and simplicity have made diode lasers one of the major actors in the laser market [1]. Consequently, increasing the output power and brightness from such devices has become a subject of great interest. Recently, single-mode diode lasers with powers in the range of $10 \mathrm{~W}$ and brightness up to $1 \mathrm{GW} / \mathrm{cm}^{2} / \mathrm{sr}$ have been developed in the near infrared [2]. Limitations to a further rise of the brightness have been related to either device failure or beam deterioration under high operating currents. The favored approach to overcome these limitations and scale up the brightness of solid-state lasers, and in particular diode lasers, is to use several moderatepower high-brightness lasers and to combine them in one single beam. During the past decade, various beam combination techniques ranging from wavelength combining to passive and active phase locking have been successfully applied to fiber lasers, slab lasers, and diode lasers, demonstrating high powers together with high efficiencies [3] . The main drawback of wavelength combining is the inherently broad spectrum, which is composed of one laser line per emitter. This disqualifies this solution for narrow absorption line optical pumping or second-harmonic generation, thereby reducing its range of applications. On the other hand, a narrow spectrum emission can be achieved with phase-locking techniques. In this work, we focus on the passive phase locking of diode lasers in an external cavity. Compared to active phase locking, this approach benefits from superior simplicity due to the self-organization process, and it does not require any additional electronic servoloop.

In the literature, two different configurations of extendedcavity passive phase locking have been extensively investigated to produce highly coherent emission from an array of diode lasers: self-imaging cavities based on the Talbot effect [4-6] and angular filtering [ $[\underline{7}, \underline{8}]$. Both approaches have demonstrated output powers in the watt range. However, array brightness has been limited by several factors: (i) the freerunning operation of the emitters, which limits the operating current range for efficient phase locking [5] ; (ii) the use of intracavity filters and magnification optics, which introduced additional losses [4]; or (iii) the beam quality of the constituent single emitters. Moreover, the spectrum is usually very unstable, which may contribute to the coherence degradation observed at high operating currents. Wavelength stabilization of the laser array can be achieved with a standard diffraction grating [ 6 ], but with the drawback of complexity. With the aim of building compact and simple cavities, we propose to use the properties of volume Bragg gratings (VBGs) to induce the stable coherent operation of arrays of diode lasers [9-11]. In this paper, we revisit the concepts of Talbot cavities and angular-selective feedback cavities, using a VBG either to induce a spectral selectivity for the Talbot cavity or to perform spatial filtering for the angular-selective feedback cavity.

The paper is organized as follows: we begin by describing the theoretical modeling of the operation of an array of singletransverse-mode lasers in an external cavity. This general model is used to describe the behavior of the two externalcavity configurations that we have investigated. Furthermore, in the case of the Talbot cavity, an iterative numerical simulation of the beam propagation within the semiconductor gain medium has been added. This allows the calculation of the near-field and far-field profiles of the phase-locked array and determination of the gain repartition inside the laser array. In the next part of this paper, we describe the experimental evaluation of the coherent combining of arrays of diode tapered lasers (TLs) of different geometries with a VBG in 
both the Talbot and the angular-filtering extended-cavity configurations. We demonstrate the benefits of using a VBG, which are derived from its spectral and spatial filtering properties.

\section{NUMERICAL MODELING OF THE EXTERNAL-CAVITY OPERATION}

\section{A. General Description of the Modal Properties of External Cavities}

We adopt the common coupled-mode analysis based on the calculation of the eigenmodes of the laser equation to evaluate the optical-field profiles of the $N$ transverse modes of an array of $N$ single-mode lasers in an external cavity, similar to the work in $[\underline{12}, 13]$. Additionally, we will take spatial filtering components inside the external cavity into account. The model will be used to predict the modal behavior of two externalcavity configurations: the Talbot external cavity and the angular-filtering cavity. In each case, the phase and intensity profiles of the array supermodes will be calculated, which depend on effective coupling between the lasers. These two external cavities are based on different filtering processes. While the Talbot cavity uses a pure diffractive near-field effect, the angular-filtering cavity operates in the far field. Finally, the angular-filtering properties of VBGs, either in transmission or reflection, will be introduced in the modeling.

We consider an array of $N$ single-mode emitters, equally spaced along the $x$ axis with a pitch $p$, as defined in Fig. 1 . All emitters are assumed to be identical, with a length $\bar{L}$ and a Gaussian transverse mode profile characterized by a full-width at $1 / e^{2}$ of $2 w$. They all have the same net optical gain $g$ and phase $\phi$. The amplitude reflectivity of the rear facet is $r_{0}$, and the front facet is antireflection coated. The external cavity, with a length $L_{\text {ext }}$, is closed by an output coupler with an amplitude reflectivity of $r$. Laser emission occurs between the rear facet of the laser bar and the output mirror of the external cavity. No free-running operation of the laser bar itself is considered in this model. We define the optical electric field vector at the front facet of the array as $\vec{E}(x, z=0)=$ $\left[u_{1}(x), u_{2}(x), \ldots, u_{N}(x)\right]$, whose components are the $N$ complex amplitudes $u_{n}(x)$ of the optical fields of each emitter $(1 \leq n \leq N)$ at $z=0$. The laser modes that may oscillate in the external cavity are those that remain identical after one round trip through the laser cavity. They are solutions of the steady-state laser equation:

$$
r_{0} r e^{2 i \varphi} e^{2 g L}\left\{\kappa_{\mathrm{km}}\right\} \times \vec{E}(x, z=0)=\vec{E}(x, z=0),
$$

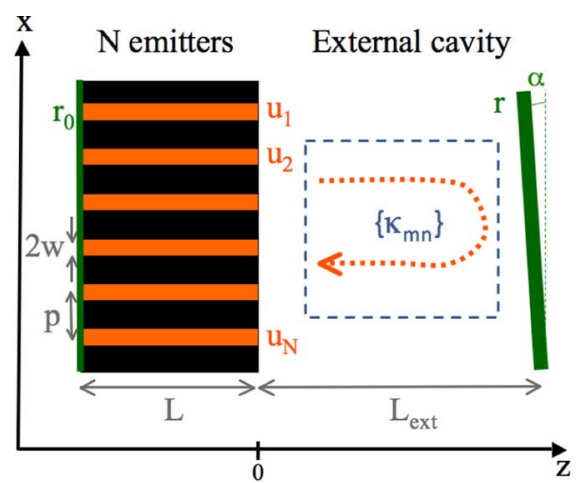

Fig. 1. (Color online) Scheme of the external cavity considered for the simulations. where $\left\{\kappa_{k m}\right\}$ is the coupling matrix of the external cavity. $\left\{\kappa_{k m}\right\}$ is a $N \times N$ matrix, whose $(k, m)$ coefficient is the coupling factor from emitter $m$ to emitter $k$ and is defined as the normalized overlap integral of the emitted field from emitter $k$ with the field emitted by emitter $m$ and backcoupled by the external cavity:

$$
\kappa_{k m}=\frac{\int_{-\infty}^{+\infty} u_{k}^{*}(x) \times C\left[u_{m}\right](x) \mathrm{d} x}{\int_{-\infty}^{+\infty} u_{k}^{*}(x) \times u_{k}(x) \mathrm{d} x}
$$

The operator $C\left[u_{m}\right]$ models the feedback from the external cavity for emitter $m$. It takes into account the free-space propagation within the external cavity and possibly any angular or spatial filter included in the external cavity. The solutions to Eq. (1) are the $N$ eigenmodes of the coupling matrix $\left\{\kappa_{k m}\right\}$. The eigenvalue $\gamma_{n}$ is proportional to the effective externalcavity reflectivity for the associated $n$th eigenmode, so the net optical gain at threshold of any of these eigenmodes is

$$
g_{n}=\frac{1}{L} \ln \left(\frac{1}{r_{0} r\left|\gamma_{n}\right|}\right) .
$$

The mode with the highest eigenvalue $\left|\gamma_{n}\right|$ has the lowest threshold and will be favored by the external cavity.

If the coupling occurs between adjacent emitters only, Eq. (1) can be solved analytically. The optical-field profiles of the $\bar{N}$ eigenmodes of a bar of $N$ Gaussian emitters ( $w$ being their half-width at $1 / e^{2}$ ) is given by

$$
\begin{aligned}
E_{n}(x, z=0)= & \sum_{k=1}^{N} \sin \left(k \frac{n \pi}{N+1}+\frac{n \pi}{2}\right) \\
& \times \exp \left(\frac{x+\left(\frac{N+1}{2}-k\right) p}{w}\right)^{2}, \quad 1 \leq n \leq N,
\end{aligned}
$$

following the approach proposed by Butler et al. [14]. The supermode $n=1$ is the in-phase mode, and the supermode $n=N$ is the out-of-phase mode. The corresponding far-field profiles consist of narrow peaks spaced by $\lambda / p$, with a fullwidth at half-maximum (FWHM) $\sim \lambda / N p$, within the envelope of the far-field pattern of the individual emitters. The number of peaks depends thus on the filling factor $2 w / p$ of the laser bar: the higher the filling factor, the lower the number of peaks.

\section{B. Properties of Volume Bragg Gratings}

Volume Bragg gratings are widely used in simple externalcavity configurations for the wavelength locking and spectral narrowing of diode laser emission-for both single emitters and arrays [15]. A few papers have demonstrated spatial beam improvement by taking benefit of their angular-filtering properties $[\underline{10}, \underline{15}]$. We have recently demonstrated experimentally that these components can also be used for the phase locking of an array of diode lasers [16]. Because of the intrinsic angular selectivity of a VBG, the use of this component inside an external cavity may modify the beam propagation and reduce the effective coupling coefficient. In order to model their effects on the modal selectivity of an external cavity, the specific reflectivity and transmission of the Bragg grating has to be taken into account. For the sake of simplicity and without loss of generality, we have considered here gratings with 

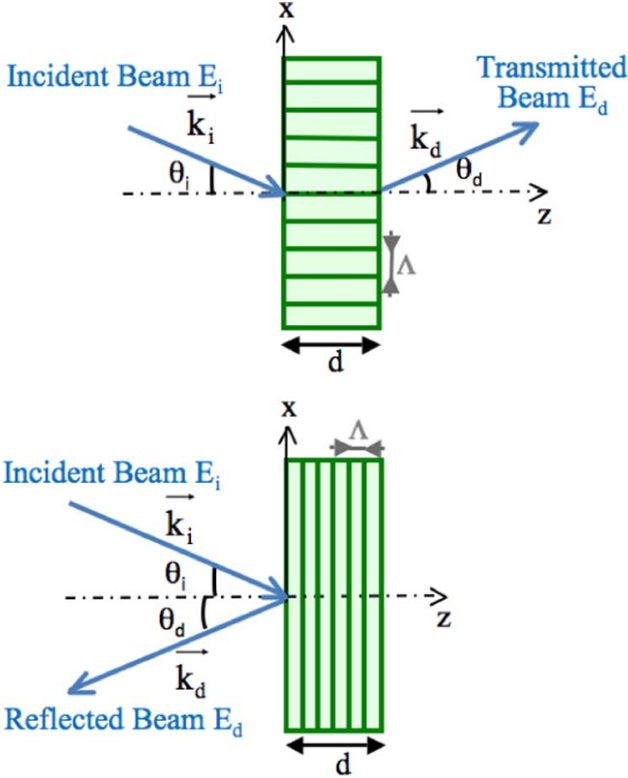

Fig. 2. (Color online) Design parameters of a transmissive (top) and a reflective (bottom) VBG; $k_{i}$ and $k_{d}$ are the direction vectors of the incident and diffracted beams, respectively; other parameters are defined in the text.

fringes perpendicular (respectively, parallel) to the optical axis for the reflective (respectively, transmissive) VBG. With this assumption, it is straightforward from [17] to write the expression of the amplitude reflectivity of a reflective VBG, with the design parameters of the gratings defined in Fig. 2. $\theta_{i}$ and $\theta_{d}$ are the incident and diffracted directions, $d$ is the thickness of the VBG, $\Lambda$ is the grating period, $n_{0}$ is the refractive index of the material, and $n_{1}$ is the modulation amplitude of the refractive index:

$$
r_{\mathrm{RBG}}\left(\lambda, \theta_{i}\right)=\frac{i}{\frac{\xi_{R}}{\nu}+\sqrt{1+\frac{\xi_{R}^{2}}{\nu^{2}}} \times \cot \left(\sqrt{\nu^{2}+\xi_{R}^{2}}\right)} .
$$

The simplified amplitude transmission of a transmissive VBG is

$$
t_{\mathrm{TBG}}\left(\lambda, \theta_{i}\right)=-i \frac{\exp \left(-i \xi_{T}\right) \times \sin \left(\sqrt{\nu^{2}-\xi_{T}^{2}}\right)}{\sqrt{1-\frac{\xi_{T}^{2}}{\nu^{2}}}},
$$

where $\xi_{R}$ and $\xi_{T}$ characterize the mismatch to the Bragg condition and are equal to

$$
\xi_{R}=\frac{\left(\frac{2 \pi}{\Lambda} \cos \theta_{i}-\frac{\pi}{n_{0} \lambda}\right) \mathrm{d}}{2 \cos \theta_{i}},
$$

$$
\xi_{T}=\xi_{R}-\frac{\pi d}{\lambda}\left(\tan \theta_{i}+1\right),
$$

and $\nu$ imposes the maximum diffraction efficiency:

$$
\nu=\frac{\pi n_{1} d}{\lambda \cos \left(\theta_{i}\right)}
$$

In this paper, we will consider VBGs designed at $\lambda_{B}=$ $975 \mathrm{~nm}$ with the specifications given in Table 1 . Their diffraction efficiencies $\left(R_{B}, T_{B}\right)$ and angular acceptances $2 \Delta \theta_{\mathrm{VBG}}$ (FWHM) roughly correspond to the gratings used in our experiments.

\section{Talbot External Cavity}

The Talbot effect is a purely diffractive phenomenon related to the propagation of an infinite coherent periodic field. It results in the appearance of self-images (in amplitude and phase) of the initial field every integer multiple of the Talbot distance $Z_{T}=2 p^{2} / \lambda$, where $p$ is the period of the field and $\lambda$ is the wavelength. Imagelike patterns also appear at fractional Talbot distances $Z_{T} / k$. In particular, the out-of-phase $(n=N)$ mode is exactly self-imaged at $z=Z_{T} / 2$. At the same distance $\left(z=Z_{T} / 2\right)$, the in-phase mode $(n=1)$ is self-imaged with a lateral shift of $p / 2$. This results in the maximum spatial discrimination of these two modes. This effect can be used in an external cavity to induce the coherent operation of a laser bar [4]. For arrays with finite dimensions, the Talbot effect can also be observed, but edge losses appear that slightly degrade the imaging quality.

\section{Coupled-Mode Analysis of the External Cavity}

In the following, we describe numerical results obtained with an array of $N=10$ emitters, with a pitch of $p=100 \mu \mathrm{m}$. The emitters have a Gaussian-shaped beam with a half-width waist at $1 / e^{2}$ of $w=15 \mu \mathrm{m}$ and the laser wavelength is $\lambda=975 \mathrm{~nm}$. The coupled-mode analysis described above was applied to the description of the external cavity, which consisted of either a planar output mirror or a reflective VBG positioned at a distance $L_{\text {ext }}$ from the bar and tilted with an angle $\alpha$ relative to the bar facet. Because we are only interested in the modal discrimination of the cavity, the reflectivity of the rear mirror and the maximum reflectivity of the external mirror were both chosen to be equal to unity $\left(r_{0}=r=1\right)$. In this case, the external-cavity operator $C$ consists of the free-space propagation within the external cavity over a distance $2 L_{\text {ext }}$. It takes into account the linear phase shift induced by the angled reflection on the output coupler, and (if appropriate) the reflectivity of the Bragg grating, which introduces an angular filtering of the optical field. The external-cavity operator was determined from the optical field after one round trip in the external cavity using the Fresnel diffraction formalism [18]:

Table 1. Design Parameters of the Volume Bragg Gratings Used for Modeling ${ }^{a}$

\begin{tabular}{cccccccc}
\hline Grating & $\theta_{B}$ & $d$ & $n_{0}$ & $n_{1}$ & $\Lambda$ & $R_{B} / T_{B}$ & $2 \Delta \theta_{\mathrm{VBG}}$ \\
\hline RBG1 & $0^{\circ}$ & $0.7 \mathrm{~mm}$ & & $3.3 \times 10^{-4}$ & $325 \mathrm{~nm}$ & $R_{B}=40 \%$ & $2.5^{\circ}$ \\
TBG1 & $0.9^{\circ}$ & $2.3 \mathrm{~mm}$ & 1.50000 & & $2.0 \times 10^{-4}$ & $20 \mu \mathrm{m}$ & $T_{B}=99 \%$ \\
\hline
\end{tabular}

${ }^{a} \theta_{B}$, Bragg angle for $\lambda_{B}=975 \mathrm{~nm}$; d, grating thickness; $n_{0}$, optical index; $n_{1}$, index modulation; $\Lambda$, grating period; $R_{B} / T_{B}$, diffraction efficiency; $2 \Delta \theta_{\mathrm{VBG}}$, FWHM angular acceptance. 


$$
\begin{aligned}
& C\left[u_{m}\right](x)=\int_{-\infty}^{+\infty} r_{\mathrm{RBG}}(\lambda, \arcsin (\sigma \lambda)-\alpha) \times H\left(L_{\mathrm{ext}}, \sigma\right) \\
& \quad \times \tilde{u}_{m}\left(\sigma-\frac{\tan 2 \alpha}{\lambda}, z=L_{\mathrm{ext}}\right) \times e^{2 i \pi \sigma} \mathrm{d} \sigma
\end{aligned}
$$

where $\tilde{u}_{m}(\sigma, z)=\int_{-\infty}^{+\infty} u_{m}(x, z) e^{-2 i \pi \sigma x} \mathrm{~d} \sigma$ is the Fourier transform of the optical field $u_{m}(x, z)$ at the distance $z$. In the Fresnel diffraction approximation, the wave propagation operator is $H(z, \sigma)=\exp \left(i \frac{2 \pi}{\lambda} z \sqrt{1-\lambda^{2} \sigma^{2}}\right)$, where $\sigma$ is the spatial frequency in the Fourier domain [18]. The demonstration of Eq. ()ㅡㄹ is detailed in Appendix A.

The coupling matrix $\left\{\kappa_{k m}\right\}$ of the Talbot external cavity was calculated with either a planar external-cavity mirror or a VBG, for various cavity round trip lengths $2 L_{\text {ext }}$ and mirror tilts $\alpha$. In all cases, the coupling coefficient between nonadjacent emitters decreased strongly as the distance between the emitters increased. Consequently, the mode profiles were very similar to the ones calculated from the weak-coupling analysis in Eq. (4). The $n=1$ supermode corresponds to the in-phase near-field profile, whereas the $n=N=10$ corresponds to the out-of-phase operation of the laser bar. With a planar output mirror, the in-phase and out-of-phase modes had the same effective coupling $\left|\gamma_{n}\right|$ at $2 L_{\text {ext }}=Z_{T}$ and $\alpha=0$, as expected. This means that the cavity provides no discrimination between these modes. Conversely, the discrimination between these two modes was a maximum for a round trip external-cavity length of $2 L_{\text {ext }}=Z_{T} / 2$, when $\left|\gamma_{N}\right|=92 \%$ and $\left|\gamma_{1}\right|=0$ (see Fig. 3). This is a consequence of the nearly perfect self-imaging of the out-of-phase mode after a $Z_{T} / 2$ freespace propagation. When the output mirror was tilted by $\alpha=\lambda / 2 p$, the coupling coefficients of the in-phase and out-of-phase modes were inverted, and $\left|\gamma_{1}\right|=86 \%$ in our simulations at $2 L_{\text {ext }}=Z_{T} / 2$. This is almost equal to $\left|\gamma_{N}\right|$ in the untilted configuration. Actually, as can be seen from Fig. $\underline{3}$, the lowest modal discrimination at $2 L_{\text {ext }}=Z_{T} / 2$ was between the $n=1$ and the $n=2$ modes for the tilted mirror and between the $n=N$ and $n=N-1$ modes for the untilted case. It was about $0.6 \mathrm{~dB}$ in both cases. Finally, the sensitivity of the coupling coefficient to adjustments of the cavity length and mirror angle was evaluated. The in-phase mode dominates the $n=2$ mode over a range of $\Delta\left(2 L_{\text {ext }}\right)=0.1 \times Z_{T}$ and $\Delta \alpha / \alpha \cong 1 \%$, which is comparable to the estimation from [19].

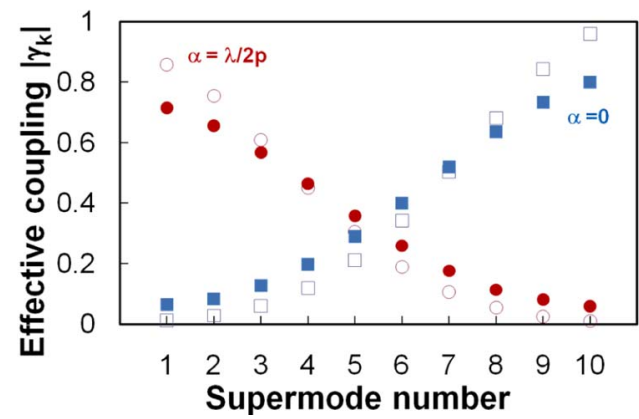

Fig. 3. (Color online) Effective coupling coefficients of the Talbot external cavity considering a plane mirror (unfilled markers) and the VBG RBG1 (filled markers) at $L_{\text {ext }}=Z_{T} / 4$ : blue squares, $\alpha=0$; red circles, $\alpha=\lambda / 2 p$. The laser array consists of $N=10$ Gaussianshaped $(w=15 \mu \mathrm{m})$ emitters, with a pitch $p=100 \mu \mathrm{m}$. The values obtained with the VBG have been normalized with respect to its maximum reflectivity $\left(R_{B}=40 \%\right)$ for easier comparison.
More interestingly, with a VBG as the external mirror, the behavior of the Talbot external cavity remains similar to the one obtained with a planar mirror. We can still define the inphase mode in which all the $N$ emitters are in phase, which is favored for the external-cavity round trip length $2 L_{\mathrm{ext}}=0.55 \times$ $Z_{T}$ and an angled Bragg grating with $\alpha=\lambda / 2 p$. The slightly longer external cavity is the result of the distributed reflectivity of the VBG. With the VBG considered here (RBG1), whose angular acceptance is twice as wide as the bar divergence, the effective coupling coefficient $\left|\gamma_{1}\right|$ of the in-phase mode is $70 \%$, and the discrimination between the $n=1$ and $n=2$ modes reduces to $0.4 \mathrm{~dB}$ (see Fig. 3). From our simulations, we predict that the coupling coefficient remains above $60 \%$ as long as the angular acceptance of the grating $2 \Delta \theta_{\mathrm{RdB}}$ is larger than the FWHM divergence of the laser bar. There is no significant change in either the near-field intensity profile or in the farfield angular distribution (see Fig. 4). In conclusion, it appears that the modal behavior of the Talbot cavity is not significantly altered with a properly chosen VBG.

It is noteworthy that only the ideal case of identical TL emitters has been considered here. A more realistic assumption would be to consider random phase differences between the emitters, resulting, for example, from differential thermal changes of the refractive index or lengths of the emitters. This would indeed have a major impact on the coherence of the external-cavity laser array. We have used a Monte Carlo statistical analysis, which attributes random phases $\left[\varphi_{1} \ldots \varphi_{N}\right]$ to the $N=10$ emitters with a uniform probability distribution. The modal properties of the Talbot external cavity with a tilted plane mirror at $L_{\text {ext }}=Z_{T} / 4$ have been calculated with the coupled-mode analysis, as a function of the standard deviation $\sigma_{\varphi}$ of the statistical sample of phases. It appears that as long as $\sigma_{\varphi}$ remains below $\pi / 5$, the in-phase mode is systematically selected by the external cavity and the visibility of the far-field profile remains typically above $80 \%$, in relatively
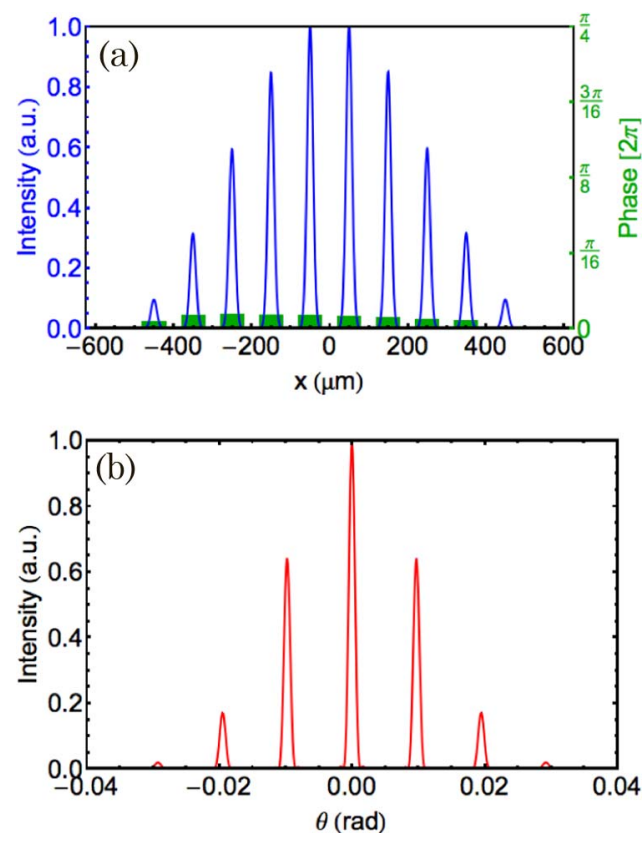

Fig. 4. (Color online) (a) Simulated near-field intensity profile (solid blue curve) and phase (green boxes) of the in-phase mode of the Talbot external cavity with RBG1 as the output coupler. Laser array as in Fig. 3. (b) Corresponding far-field angular profile. 
good agreement with the experimental results from Cassarly et al. [20] obtained with a 20-emitter external Talbot cavity and an intracavity liquid-crystal phase shifter. The deterioration of the coherence of the laser array is evidenced in the simulated far-field profiles by wider interference peaks and parasitic sidelobes. The visibility drops to $50 \%$ for $\sigma_{\varphi}$ values in the range of $\pi / 2$.

\section{Electrical and Optical Numerical Modeling of the Tapered-Laser Array}

In order to further understand the limitations of the Talbot external cavity with our diode laser bar, we have modeled the propagation of the optical beam inside the semiconductor laser array. The details of the semiconductor layers are given in [21]. The model of the tapered-laser bar combines electrical and optical models. The isothermal electrical model consists of the 1D unipolar carrier-diffusion equation [Eq. (9)], where the injection current density $J^{(k)}(x)$ is assumed to be constant inside each laser stripe and zero outside it. This equation is solved for the carrier density distribution $n^{(k)}(x)$ inside the TL $(k=1$ to $N)$ so that spatial hole burning and carrier lensing effects are included:

$$
D_{a} \frac{d^{2}}{d x^{2}} n^{(k)}(x)=-\frac{J^{(k)}(x)}{q d}+R_{\mathrm{NR}}^{(k)}(x)+R_{\mathrm{Sp}}^{(k)}(x)+v_{g} g^{(k)}(x) S(x),
$$

where $D_{a}, n^{(k)}, J^{(k)}, d, R_{\mathrm{NR}}^{(k)}, R_{\mathrm{Sp}}^{(k)}, v_{g}, g^{(k)}$, and $S$ are the ambipolar diffusion coefficient, carrier density, injection current density, active region thickness, nonradiative recombination rate, spontaneous emission rate, group velocity, optical gain, and photon density, respectively [22]. The optical fields in the individual TLs are modeled with the $2 \mathrm{D}$ wide-angle finite difference beam propagation method using the effective index approximation. As described by Lim et al. [22], the electrical model is coupled to the optical model through stimulated emission/absorption and spontaneous emission coupling, and through carrier-induced changes in the complex refractive index. Free-running emission of the TL without the external cavity is not considered here, because an antireflection coating ( $R=0.1 \%$ ) is assumed on the front facet. A planar mirror with a reflectivity of $40 \%$ is used as the output coupler for the external cavity, and, as previously, we assume that the rear facet reflectivity $r_{0}$ is unity (see Fig. 1). An iterative approach based on the Fox-Li method was used, whereby the optical field was propagated back and forth inside the cavity (semiconductor laser + external cavity) until a steady-state condition (in terms of optical power and amplitude profile) was reached. The resulting supermode profiles, either in phase or out of phase depending on the tilt of the output coupler, were actually very similar to the ones obtained from our previous simpler model assuming Gaussian emitters (Subsection 2.C.1) [23]. This can be attributed to the nearly single-transverse mode operation of the TL. Nevertheless, we observe increased propagation losses at the edges of the TL array due to the imperfect Talbot imaging with a finite array. This is obvious from the variations of the optical gain at threshold $G_{k}$ for each emitter $(k=1$ to $N)$, which is proportional to the round trip losses along the array (Fig. 5). $G_{k}$ was evaluated as the ratio between the optical power on the front facet $(z=0) \int I_{\text {out }}^{(k)}(x) \mathrm{d} x$ after one round trip inside emitter $k$ and the optical power reflected

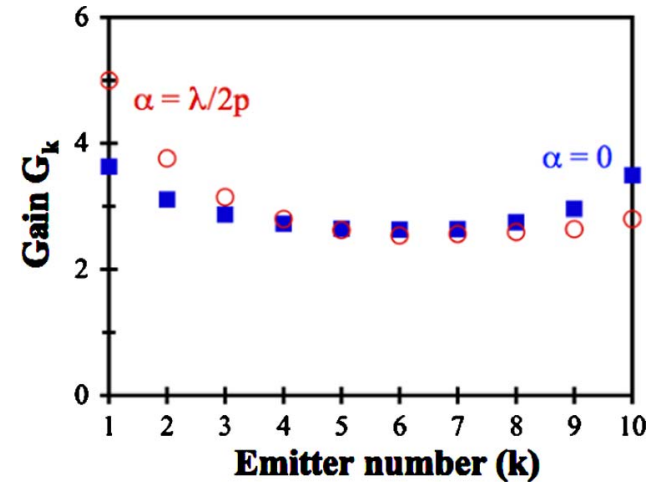

Fig. 5. (Color online) Effective gain repartition inside the laser array for the Talbot external cavity with the $N=10$ index-guided TL array: filled squares, out-of-phase mode $(\alpha=0)$; unfilled circles, in-phase mode $(\alpha=\lambda / 2 p) ; L_{\text {ext }}=Z_{T} / 4$.

by the external-cavity mirror and coupled into the same emitter $\int I_{\mathrm{in}}^{(k)}(x) \mathrm{d} x$, where $I_{\mathrm{in}}^{(k)}(x)$ and $I_{\text {out }}^{(k)}(x)$ are the respective forward and backward propagating optical intensity profiles along the width of the front facet of the emitter. Indeed, the edge emitters for the out-of-phase mode in a $Z_{T} / 4$ long untilted ( $\alpha=0$ ) external cavity have $40 \%$ greater loss than the central emitters. These additional losses reach $90 \%$ for the in-phase mode in a $Z_{T} / 4$ long tilted $(\alpha=\lambda / 2 p)$ external cavity due to the asymmetric feedback. This will lead to a lower coupling strength of the external cavity and an increased lasing threshold for these edge emitters, which may prevent their phase locking. Finally, from these simulations, we evaluate the normalized external-cavity coupling efficiencies for the in-phase and out-of-phase modes to be $77 \%$ and $82 \%$, respectively. These values are in good agreement with the values $\gamma_{1}$ and $\gamma_{N}$ deduced from the coupled-mode analysis.

\section{Angular-Filtering External Cavity}

The coupled-mode analysis of the extended-cavity laser array was also applied to the description of the angular-filtering external-cavity design, which uses angular-selective coupling between the emitters to select one array supermode. The external cavity simply consists of a mirror that reflects light from the laser bar at an angle $\theta_{0}$, on a $2 \delta \theta$ full-width angle. The supermode whose far-field profile has the highest overlap with this angular filter is selected by the cavity. Experimentally, the mirror may be a planar slit mirror in the focal plane of a Fourier lens [ㄱ, $\underline{8}]$, a cylindrical concave mirror [24] , or a VBG, either in transmission or in reflection [25]. Similar angular-filtering cavities, or asymmetric-feedback cavities, are also used with broad area single-emitter diode lasers to select a particular lateral mode [23]. The related external-cavity operator, which expresses - in the Fourier domain - the filtering induced by the external cavity on the far-field profile of each emitter, is given by

$$
C\left[u_{m}\right](x)=\int_{-\infty}^{+\infty} f(\arcsin (\lambda \sigma)) \times \tilde{u}_{m}(\sigma) e^{2 i \pi x \sigma} \mathrm{d} \sigma,
$$

with $\tilde{u}_{m}(\sigma, z)$ being the Fourier transform of the optical field $u_{n}(x, z)$, as before. The filtering function $f(\theta)$ may be a simple rectangular profile centered at $\theta_{0}$ and with a full-width $2 \delta \theta$. Alternatively, for the case described in Fig. $\underline{6}$ where only the beam diffracted by the VBG is reflected back into the laser array, it may be the two-pass Bragg grating transmission 
$t_{\mathrm{TBG}}(\lambda, \theta) \times t_{\mathrm{TBG}}^{*}(\lambda, \theta)$. The filtering direction $\theta_{0}$ is determined by the orientation of the VBG. The laser output is the part of the beam that is not diffracted by the VBG. Again, a $100 \%$ reflectivity is considered here for the feedback mirror for the sake of simplicity.

We first calculated the transverse supermodes of an external cavity using the very same bar considered in Subsection 2.C and a rectangular filter as the angular-selective mirror. These supermodes are the solutions of Eq. (1) with the external-cavity operator given by Eq. (10). The mirror width $2 \delta \theta$ was chosen as the diffraction-limited angular width of the laser array $\lambda / N p$. As expected, the in-phase mode $(n=1)$ is favored for an on-axis filter $\left(\theta_{0}=0\right)$, because it is the only mode with an axial peak in the far field. The numerical calculations show that its coupling coefficient is $\left|\gamma_{1}\right|=30 \%$. Conversely, when the filter is at an angle of $\theta_{0}=\lambda / 2 p$, the $n=$ $N=10$ mode is selected, because the angular filter overlaps one main peak of the far-field profile of the out-of-phase mode. The corresponding coupling coefficient is $\left|\gamma_{N}\right|=25 \%$. The lower effective coupling for these two modes, as compared to the modes of the Talbot external cavity, is due to the poor overlap of the multilobed far-field profiles with the rectangular filter. This results in a higher external-cavity laser threshold and makes the laser more sensitive to parasitic phase shifts.

The angular-filtering external-cavity configuration is more attractive for high-filling-factor laser bars, because the farfield profiles of the in-phase and out-of-phase modes reduce to one or two main lobes. In the simplest setup, the laser array is forced to operate in the out-of-phase two-lobe mode by using a mirror to reflect back one lobe, while using the second lobe as the output beam of the external cavity. This is the actual situation for the off-axis mirror external-cavity experiments reported in [7,24]. Here, we consider the case of a bar of $N=6$ single-transverse-mode emitters with a pitch of $p=30 \mu \mathrm{m}$ and a waist radius of $w=15 \mu \mathrm{m}$. This corresponds to our experimental configuration in Subsection 3.B. First, a rectangular angular filter centered at $\theta_{0}=\lambda / 2 \overline{p=}$ $16 \mathrm{mrad}$ with a full-width $2 \delta \theta=\lambda / N p=6 \mathrm{mrad}$ is used in the external cavity. The array supermode with the highest coupling coefficient $\left(\left|\gamma_{N}\right|=32 \%\right)$ is the out-of-phase mode. The second strongest mode is the $n=N-1=5$ supermode, and the maximum discrimination between these two modes $\left|\gamma_{6}\right| /\left|\gamma_{5}\right|$ is about $5 \mathrm{~dB}$. Secondly, the array supermodes were calculated using the transmission Bragg grating TBG1 (see Table 1) as the angular-filtering component, and a highreflection mirror $(r=1)$ on the diffracted beam (see Fig. 6). This grating was designed to diffract light around $\theta_{B}=$

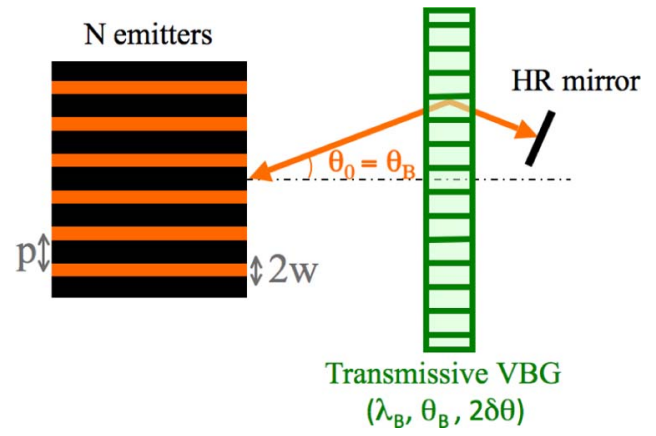

Fig. 6. (Color online) Scheme of the angular-filtering cavity considered for the simulations.
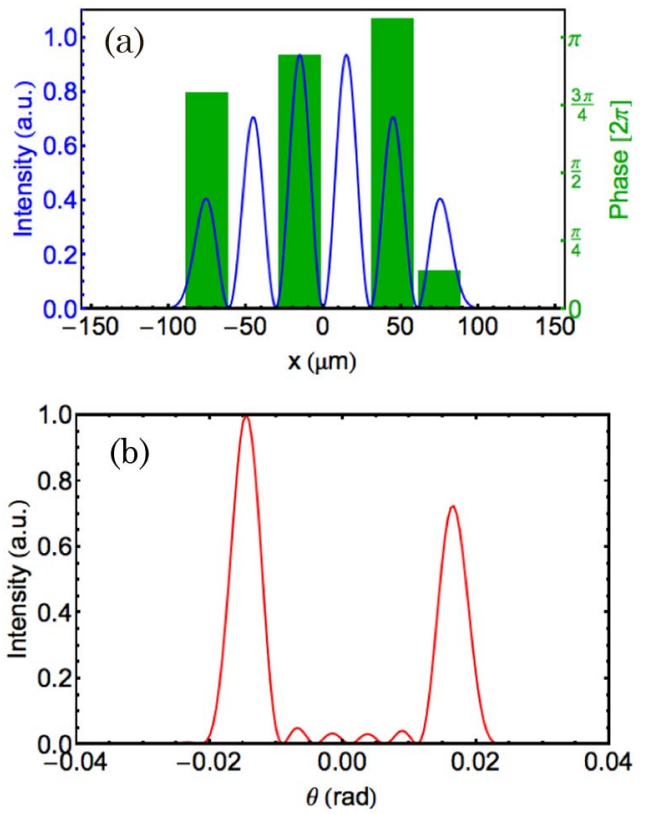

Fig. 7. (Color online) (a) Simulated near-field intensity profile (solid blue curve) and phase (full green boxes) of the out-of-phase mode of the angular-filtering cavity. The laser array consists of $N=6$ Gaussian-shaped $(w=15 \mu \mathrm{m})$ emitters, with a pitch $p=30 \mu \mathrm{m}$. (b) Corresponding far-field angular profile.

$\pm 16 \mathrm{mrad}$, with an angular acceptance $2 \delta \theta=6 \mathrm{mrad}$, similar to the rectangular filter used previously. Again, the $(N=6)$ out-of-phase supermode has the highest coupling efficiency $\left|\gamma_{N}\right| \cong 30 \%$. The phase shift between adjacent emitters is $\pi \pm 0.2 \pi$, as shown in Fig. 7 . The far-field profile is close to the one obtained with a rectangular filter (Fig. 7). This configuration of angular-filtering with such a high-filling-factor diode laser array is of great interest, because it is possible to achieve both efficient coupling and good modal discrimination together with a single-lobe output.

\section{EXPERIMENTAL VALIDATION}

In this section, we describe the experimental investigations of the passive phase locking of arrays of diode lasers using the concepts described in Section 2. We used arrays of diode TLs, because they can provide high output power together with quasi-single-transverse mode operation $\left(M^{2}<2\right)$.

\section{A. Self-Imaging Talbot External Cavity}

In the Talbot external-cavity configuration, two geometries of TL arrays were used (Table 2). The first configuration is an array of index-guided narrow-tapered diode lasers with a pitch of $p=100 \mu \mathrm{m}$. The second configuration is an array of gainguided wide TLs with a pitch of $p=500 \mu \mathrm{m}$. In each case, the beam of the whole array is collimated in the fast-axis direction by a high-numerical-aperture acylindrical lens. The cavity is closed by a VBG located at $\sim Z_{T} / 4$ from the front facet to give the maximum modal discrimination between the in-phase and out-of-phase modes (see Subsection 2.C).

The design of the array of the $N=10$ index-guided TL is described in the previous section. This laser array was provided by Alcatel Thales III-V Lab (Palaiseau, France) [21]. In the slow-axis direction, the 10 beams propagate freely inside the external cavity. A VBG with a reflectivity of $40 \%$ was placed at a quarter of the Talbot distance from the front facet 
Table 2. Geometric Properties of the Tapered-Laser Arrays Used in the Experiments

\begin{tabular}{lcccc}
\hline $\begin{array}{c}\text { Guiding } \\
\text { Type }\end{array}$ & $\begin{array}{c}\text { Number of } \\
\text { Emitters } N\end{array}$ & Pitch $p$ & $\begin{array}{c}\text { Tapered } \\
\text { Angle }\end{array}$ & $\begin{array}{c}\text { Output Facet Width } \\
\text { of Each Laser }(2 w)\end{array}$ \\
\hline Index & 10 & $100 \mu \mathrm{m}$ & $<1^{\circ}$ & $\sim 30 \mu \mathrm{m}$ \\
Index & 6 & $30 \mu \mathrm{m}$ & $<1^{\circ}$ & $\sim 30 \mu \mathrm{m}$ \\
Gain & 12 & $500 \mu \mathrm{m}$ & $6^{\circ}$ & $\sim 200 \mu \mathrm{m}$ \\
\hline
\end{tabular}

and was used as the output coupler. Thanks to the small array pitch, the external cavity was very short, $L_{\text {ext }} \cong Z_{T} / 4=5 \mathrm{~mm}$. As expected, the external cavity operated in the out-of-phase mode if the VBG is untilted. Conversely, the in-phase mode was forced by angling the VBG by $\lambda / 2 p(5 \mathrm{mrad})$. A maximum output power of $1.7 \mathrm{~W}$ was obtained for an operating current of $3.9 \mathrm{~A} \mathrm{[16]}$. The output power was only limited by the thermal rollover of the array, which appeared at $4 \mathrm{~A}$ under our experimental conditions. A major feature of our Talbot cavity setup was the use of a VBG as the output coupler to stabilize the wavelength. In order to demonstrate the beneficial effect of the VBG on the phase locking, we compare in Fig. 8 the farfield patterns obtained with a simple $R=40 \%$ planar mirror as the output coupler (a) and that obtained with a VBG with a reflectivity of $40 \%$ centered at $972 \mathrm{~nm}$ (b). These far-field patterns were measured for an operating current equal to $\sim 2.5$ times the laser threshold. In both cases, in-phase mode operation was obtained. The interference peaks in the far field are narrow, with a FWHM width below 1.4 times the diffraction limit $\lambda / N p$; this indicates that most of the emitters are actually phase locked and contribute to the coherent emission of the laser array. The envelope of the far-field profile has a total width of roughly $120 \mathrm{mrad}$, which is larger than would be expected from single-transverse-mode emitters. This was due to

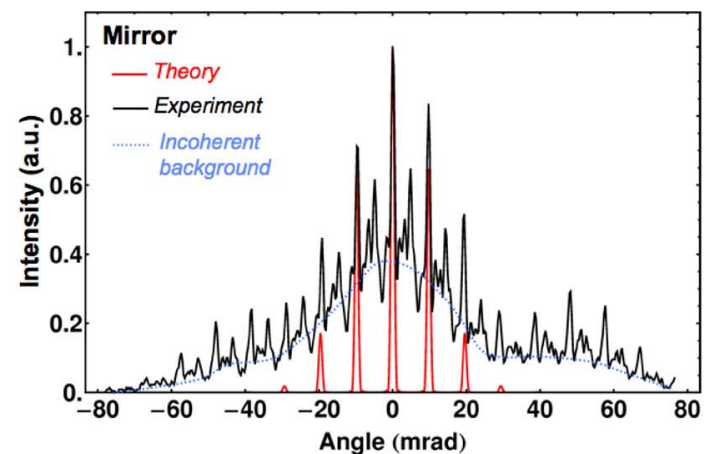

(a)

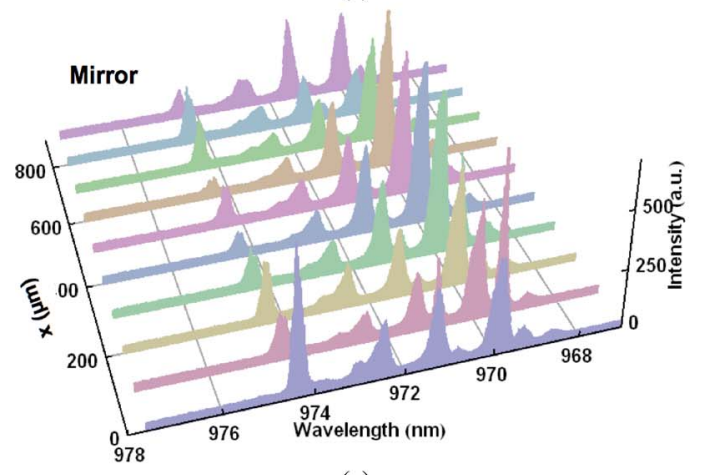

(c) a deterioration of the bar at the time of this comparative experiment, which has resulted in a slightly multimode operation of the TL characterized by an average $M^{2}$ beam quality factor of 3. However, it is obvious in Fig. 8 that the incoherent background, which consists in a broad pedestal in the far-field profile, is much stronger with the planar mirror. Intense additional sidelobes associated to competing transverse supermodes are also visible in Fig. 8(a). In order to quantify the phase-locking quality of these two far-field patterns, the fringe visibility $V=\left(I_{\max }-I_{\min }\right) /\left(I_{\max }+I_{\min }\right)$ was measured around the central peak. With the planar mirror, the visibility was $V=0.4$, but it increased to 0.8 with the VBG under the same operating conditions. From the statistical analysis of the Talbot external cavity (Subsection 2.C.1), we roughly estimate that in the first case, the standard phase deviation of the laser array is larger than $\pi / 2$, though it should remain in the range of $\pi / 5$ with the VBG. This clearly demonstrates that the use of the VBG enhanced the coherence of the TL array. This improved behavior is attributed to the reduction of the wavelength competition inside the cavity. Indeed, the measured emission spectrum of the external-cavity laser closed by the planar mirror contained more than seven laser lines over a spectral range of $6 \mathrm{~nm}$. Conversely, the use of the VBG allowed only one laser line centered at the Bragg wavelength with a spectral linewidth of $\sim 100 \mathrm{pm}$-irrespective of the operating conditions [Fig. 8(d)]. These results validate that the use of a VBG as the output coupler of the external Talbot cavity favors stable phase-locked operation of the external cavity, due to the strong spectral stabilization of the laser emission. This also permits the laser array to reach high operating currents (more than four times the threshold) with no significant degradation of the phase locking. Even better results have been already demonstrated with a VBG and the

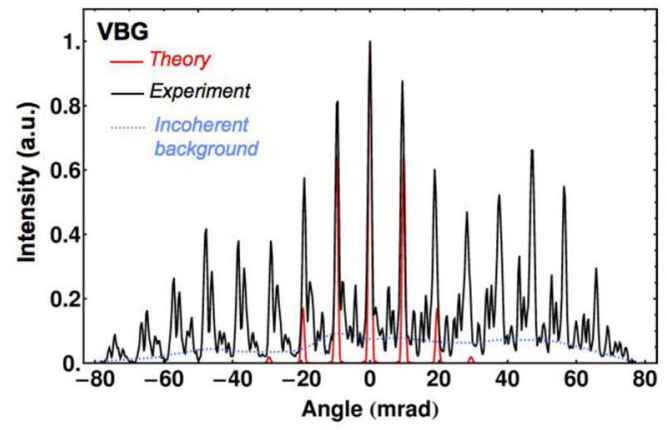

(b)

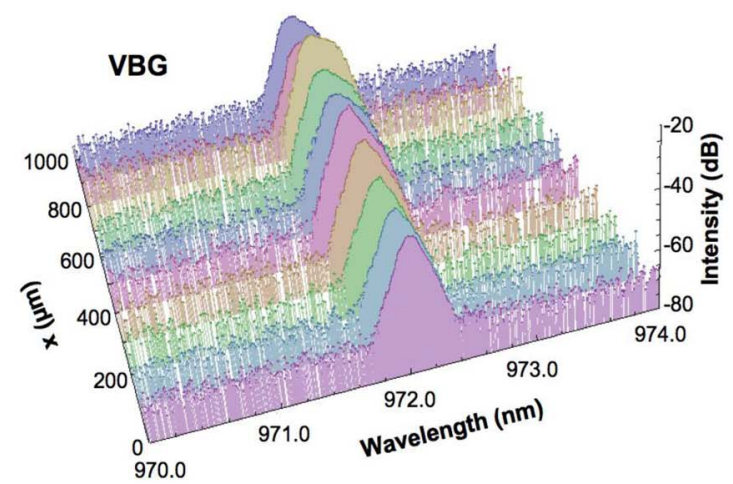

(d)

Fig. 8. (Color online) Far-field profiles of the phase-locked $N=10$ index-guided TL array (in-phase mode) in the Talbot external cavity with a $40 \%$ reflectivity plane mirror (a) or VBG (b) as the output coupler. (c), (d) Corresponding spatially resolved near-field spectra. 
same TL bar before its degradation, with a narrower far field and a visibility of $V \cong 0.9$ [16]. This makes VBGs a promising tool for increasing the output power from coherent diode laser arrays in external Talbot cavity configurations and makes it possible to overcome the usual limitation related to the incoherent background at high operating currents.

In order to further increase the output power, an array of gain-guided TLs was used. Indeed, the gain-guiding technology allows high output powers in the $10 \mathrm{~W}$ range from single emitters with no significant degradation of the beam quality $[2,26]$. The laser bar used was designed and grown at the Fraunhofer Institut für Angewandte Festkörperphysik (Freiburg, Germany). It consisted of $N=12$ tapered diode lasers similar to the one described in [26], with an $0.1 \%$ antireflection coating on the front facet. The emitters were separated by a pitch of $500 \mu \mathrm{m}$ (see Table 2), with an $0.1 \%$ antireflection coating on the front facet. The free-running bar emitted $8.9 \mathrm{~W}$ at $20 \mathrm{~A}$ in a broad spectrum centered at $983 \mathrm{~nm}$ at $T=20^{\circ} \mathrm{C}$. The laser bar was collimated along the fast axis. An additional phase plate was specifically designed to separately collimate each emitter along the slow axis and to correct the astigmatism, the fast-axis smile deformation, and pointing errors [27]. This decreased the divergence of the whole array to $15 \mathrm{mrad}$ along the slow axis and $4 \mathrm{mrad}$ along the fast axis. Furthermore, it improved the global coupling between emitters through the external-cavity feedback. A reflecting VBG $\left(R_{B}=25 \%\right.$ at $\lambda=981 \mathrm{~nm}$ ) was chosen as the output coupler of the Talbot external cavity, whose length was $Z_{T} / 4=$ $130 \mathrm{~mm}$. The longer cavity resulted from the wider pitch of the array, which is a standard choice for high-power laser bars due to thermal management considerations. Like the indexguided TL bar, the laser line was locked to the Bragg wavelength within $0.2 \mathrm{~nm}$ at $-10 \mathrm{~dB}$ over the whole operating range. The slow-axis far-field profile exhibited narrow peaks $(<1 \mathrm{mrad}$ FWHM) characteristic of the coherent operation of the whole TL array (Fig. 9), with a high visibility at threshold $(V=0.9)$. In this case, the number of peaks in the far-field profile was only seven, thanks to the collimation of the emitters in the slow-axis, which increased the filling factor to $\sim 25 \%$ as compared to the bare TL array (filling factor $\sim 1 \%$ ). The visibility $V$ of the far-field pattern decreased at higher currents as the width of the peaks increased, due to strong competition with the incoherent, free-running operation of the

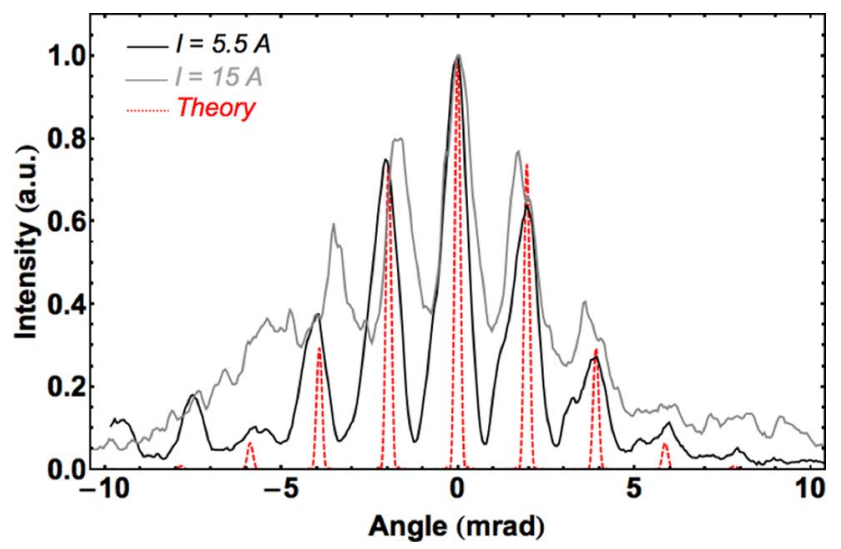

Fig. 9. (Color online) Far-field profiles of the phase-locked $N=12$ gain-guided TL array in the external Talbot cavity with a 25\%-reflectivity VBG; red dashed curve, theory; black curve, operating current $I=5.5 \mathrm{~A}$; gray curve, $I=15 \mathrm{~A}$. laser bar. At $15 \mathrm{~A}, V=0.5$ for an output power of $2.5 \mathrm{~W}$ and the peak width was $\sim 1.2 \mathrm{mrad}$. Above $20 \mathrm{~A}$, the external cavity was no longer able to phase lock the emitters.

In conclusion, we demonstrated the passive phase locking of arrays of both index-guided and gain-guided diode tapered lasers in a Talbot external cavity. The use of a VBG as the output coupler locked the bar wavelength to less than $0.2 \mathrm{~nm}$ and favored coherent phase-locked operation. The angular profile of the emission exhibited a few peaks due to the high filling factor of the bars. Power in the $2 \mathrm{~W}$ range was obtained, which is comparable to the highest power demonstrated to date from phase-locked diode lasers [5]. The loss of coherence at high operating currents is attributed to the parasitic free-running lasing of the individual emitters. This is revealed by a decrease of the far-field visibility related to an increased incoherent pedestal. In the case of the index-guided TL bar, phase-locked operation was characterized both by the high visibility of the far-field profile and the narrowness of its interference lobes, which were close to the diffraction limit $\lambda / N p$. This indicates that the entire bar efficiently contributed to the phase-locked laser emission. The experimental setup used was similar in principle to the one described by Huang et al. [5] with a bar of 10 single-transverse mode lasers [slab-coupled optical waveguide lasers (SCOWLs)] and a pitch of $p=100 \mu \mathrm{m}$. The most notable difference was the use of a VBG in our extended cavity. However, the lower filling factor of the SCOWL array resulted in a broader far-field emission and a larger number of peaks. Additionally, the wider far-field peaks (as compared to the diffraction-limited divergence) demonstrated that some emitters were not properly phase locked. Nevertheless, the fact that Huang et al. have demonstrated a higher power makes us confident that better results would be obtained with improved index-guided TLs. In the case of the high-power, gain-guided tapered-laser bar, the Talbot external-cavity setup was more complex, with corrective optics and a long cavity because of the wider pitch of the array. The very high gain of these emitters makes it difficult to avoid the incoherent free-running emission of the individual emitters, which becomes predominant at high operating currents. This reduces the coherence of the phase-locked mode, as evidenced by the low visibility $V$ of the far-field fringes and their increased width, which reaches seven times the diffraction limit $\lambda / N p$. Nevertheless, gain-guided TLs are capable of a very highpower and high-brightness emission. They are very promising for future experiments, as a reduction of the front facet reflectivity can be obtained by means of an improved antireflective coating or an angled facet.

\section{B. Angular-Filtering External Cavity}

As discussed in Subsection 2.D, the angular-filtering technique may be of particular interest in the context of high-fillingfactor arrays, because the coherent far-field profile has a very simple structure with only one or two peaks (see Fig. 7). A diffraction-limited single-lobe beam is obtained by selecting the out-of-phase mode with the angular filter positioned on one of its two major lobes. The laser output is then collected on the symmetric lobe. The experimental setup is described in Fig. 10. We used a laser array containing six index-guided TL, each identical to the ones used in the first Talbot cavity (Table 2). The pitch was $30 \mu \mathrm{m}$, so the filling factor of this array was close to $100 \%$. The laser beam was collimated in the 


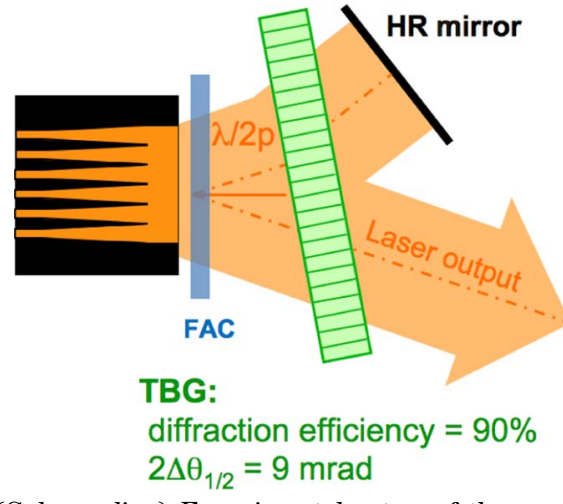

Fig. 10. (Color online) Experimental setup of the angular-filtering cavity; FAC, fast-axis collimator; TBG, transmissive VBG.

fast-axis direction by a high-numerical-aperture acylindrical lens. As the angular filter, we used a transmissive VBG with a diffraction efficiency of $90 \%$ at $980 \mathrm{~nm}$ and an angular acceptance of 9 mrad. Out-of-phase mode operation is obtained by tilting the VBG to diffract the beam emitted in the direction $\theta=\lambda / 2 p$, which corresponds to the angular location of one of its two major lobes. This beam is then reflected back into the laser array by a highly reflective mirror $(R>90 \%)$. The laser output located on the symmetric angular direction $(-\lambda / 2 p)$ is not diffracted by the VBG, because it does not match the Bragg condition. Figure 11 shows the far-field pattern obtained for an operating current of $2.3 \mathrm{~A}$. It clearly exhibits one major peak located close to $\lambda / 2 p=16 \mathrm{mrad}$, confirming the out-of-phase mode operation. On the symmetric angular direction $(-\lambda / 2 p)$, a small gap can be seen due to the angular components diffracted by the VBG. In this configuration, a maximum output power of $1.3 \mathrm{~W}$ has been obtained for an operating current of $3 \mathrm{~A}$. The output beam has a width of $7.5 \mathrm{mrad}-1.4$ times the diffraction limit $\lambda / N p$ - and it contains $33 \%$ of the optical power.

A similar preliminary experiment was done with a highly reflective VBG tilted to reflect light in the direction $\lambda / 2 p$ [25]. The VBG reflectivity was $99 \%$ at $979 \mathrm{~nm}$, its spectral bandwidth was $0.3 \mathrm{~nm}$, and its angular acceptance was $35 \mathrm{mrad}$. In this configuration, coherent operation in the out-of-phase mode was observed with a $7.5 \mathrm{mrad}$ wide single lobe in the far-field profile. Because of the rather large angular acceptance of the VBG compared to the diffraction limit of

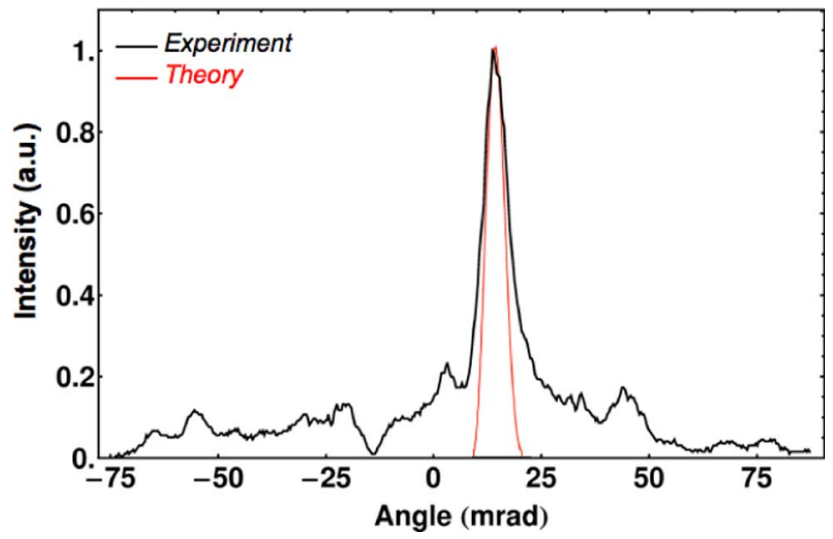

Fig. 11. (Color online) Experimental far-field profile of the phaselocked $N=6$ index-guided TL array in the angular-filtering cavity with a transmissive VBG; operating current, $I=2.3 \mathrm{~A}$; output power, $P=971 \mathrm{~mW}$ the laser array, the mode competition was high. This added to the instability of the cavity at high output powers. Nevertheless, thanks to the narrow spectral acceptance of the VBG, the spectrum of the whole array was locked to the Bragg wavelength within $0.15 \mathrm{~nm}$ at $-3 \mathrm{~dB}$. In both configurations, it was noteworthy that the angular width of the TL array emission roughly corresponded to the diffraction limit $(\sim 7 \mathrm{mrad})$ of the whole array. This was a clear proof that the narrow far-field profile resulted from the coherent combining of the TL emitters and not from an incoherent addition of filtered beams.

\section{CONCLUSION}

Coupled-mode analysis is a powerful tool to describe and understand the spatial properties of an array of single-mode emitters in an external cavity, such as its near- and far-field profiles or the coupling efficiency and the discrimination between competing modes. It has been applied here to model two different configurations, the self-imaging Talbot external cavity, which is intrinsically a near-field filtering process, and the angular-filtering cavity, which works in the far field. Furthermore, we have introduced advanced filtering functions in the modeling of the external cavity, as the transmission or the reflection of a VBG. It is worth noting that the same approach could be applied to other setups as the self-Fourier cavity [28] or external cavities with diffractive optical elements [29]. In the two configurations studied here, we have demonstrated theoretically that VBGs may be used as filtering components without loss of selectivity of the external cavity. This model has enabled us to establish the modal behavior as well as the sensitivity of the cavities to misalignments.

Experimental investigations were also made of both cavities In the case of the angular-filtering cavity, a transmissive VBG was used to selectively feed back one of the two peaks of the out-of-phase mode located at $\theta=\lambda / 2 p$. A single-lobe output, in the symmetrical direction is then obtained with a beam close to the diffraction limit. In the case of the Talbot cavity, the beneficial effect of the spectral stabilization by the VBG on the phase locking was demonstrated. The incoherent freerunning emission of the laser array was significantly reduced. This made it possible to operate the laser array up to high pump currents and to reach output powers in the watt range, with still highly contrasted fringes in the far-field profile $(V>50 \%)$, which is evidence of the strong coherence of the laser array. Additionally, the VBG forces the laser emission into a wavelength-stabilized narrow spectrum $(\Delta \lambda<0.2 \mathrm{~nm})$; thus, the spectral brightness of the TL bars is significantly improved. This is an obvious benefit of our configuration for applications requiring a high spectral purity laser source, such as nonlinear frequency conversion or pumping of solid-state lasers on narrow absorption lines. Two different kinds of tapered diode laser bars were used, with different pitches $p$ and laser cavity designs. On the one hand, the $p=100 \mu \mathrm{m}$ indexguided TL bar resulted in a short external cavity, which is favorable for stable operation. Nevertheless, the output power was limited by the laser bar itself. On the other hand, the wide gain-guided TL bar can deliver very high output powers, but the inherent astigmatism of the emitters as well as the lateral smile of the bar had to be corrected. Furthermore, in our preliminary experiments, the phase-locked operation of the TL bar was limited by its free-running emission. Thanks to the 
intrinsic good beam quality of both TL designs, high spatial brightness of the coherent emission of the phase-locked arrays was demonstrated; the simultaneous narrowing and stability of the spectrum was induced by the VBG. Many applications which require these three features would benefit from any further improvement of these results.

\section{APPENDIX A}

The different steps to obtain the expression of the cavity operator $C\left[u_{n}\right]$ for the Talbot cavity [Eq. ()] ] are described in Fig. 12; they follow the free-space beam propagation on a round trip inside the external cavity and its reflection on a tilted volume Bragg mirror. The optical fields are expressed within the framework of the Fresnel diffraction theory.

The first step is the free-space propagation of the optical field inside the external cavity on the distance $L_{\text {ext }}$. Using the Fresnel approximation, the expression of the optical field $u_{n}\left(x, z=L_{\mathrm{ext}}\right)$ is straightforward [18]:

$$
u_{n}\left(x, z=L_{\mathrm{ext}}\right)=\int_{-\infty}^{+\infty} H\left(L_{\mathrm{ext}}, \sigma\right) \times \tilde{u}_{n}(\sigma, z=0) e^{2 i \pi x \sigma} \mathrm{d} \sigma,
$$

where $\tilde{u}_{n}(\sigma, z=0)=\int_{-\infty}^{+\infty} u_{n}(x, z) e^{-2 i \pi \sigma x} \mathrm{~d} \sigma$ is the Fourier transform of the optical field $u_{n}(x, z=0), H(z, \sigma)=$ $\exp \left(i \frac{2 \pi}{\lambda} z \sqrt{1-\lambda^{2} \sigma^{2}}\right)$ is the wave propagation operator, and $\sigma$ is the spatial frequency in the Fourier domain.

The second step is the reflection of the tilted VBG. Its angular selectivity is taken into account by multiplying each angular component of the optical beam by $r_{\mathrm{RBG}}$ [as given in Eq. (5)], which has to be done in the Fourier domain. Moreover, the tilt of the VBG adds a linear phase shift $\varphi(x)=$ $2 \pi x \tan (2 \alpha) / \lambda$ to the optical field, corresponding to a shift of $\tan (2 \alpha) / \lambda$ in the Fourier domain. The resulting optical field in the Fourier domain is then given by

$$
\begin{aligned}
\tilde{u}_{n}\left(x, z=L_{\mathrm{ext}}, \alpha\right)= & r_{\mathrm{RBG}}(\lambda, \arcsin (\sigma \lambda)-\alpha) \\
& \times \tilde{u}_{n}\left(\sigma-\frac{\tan (2 \alpha)}{\lambda}, z=L_{\mathrm{ext}}\right),
\end{aligned}
$$

where the angular argument in $r_{\mathrm{RBG}}$ is corrected by $\alpha$, because the angular reference in Eq. (5) is perpendicular to the grating interface.

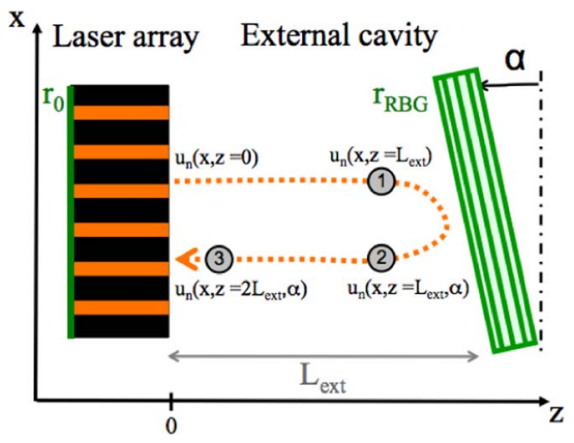

Fig. 12. (Color online) Scheme of the external cavity with parameters used for the demonstration of Eq. () (see details in Appendix A).
Finally, the last step is the free-space propagation of the field back to the laser array, which is written with the Fresnel integral similarly to Eq. (A1):

$$
\begin{aligned}
u_{n}\left(x, z=2 L_{\mathrm{ext}}, \alpha\right)= & \int_{-\infty}^{+\infty} H\left(L_{\mathrm{ext}}, \sigma\right) \\
& \times \tilde{u}_{n}\left(\sigma, z=L_{\mathrm{ext}}, \alpha\right) e^{2 i \pi x \sigma} \mathrm{d} \sigma .
\end{aligned}
$$

As $C\left[u_{n}(x)\right]=u_{n}\left(x, z=2 L_{\text {ext }}, \alpha\right)$, this leads eventually to Eq. () for the external-cavity operator.

\section{ACKNOWLEDGMENTS}

This work was supported within the European project WWW .BRIGHTER.EU under grant FP6-IST-035266. D. Pabœuf thanks the French Ministry of Defense for the funding of his Ph.D.

\section{REFERENCES AND NOTES}

1. G. Overton, S. G. Anderson, D. A. Belforte, and T. Hausken, "Laser marketplace 2010: how wide is the chasm?" Laser Focus World 46, 32-49 (2010).

2. C. Fiebig, G. Blume, C. Kaspari, D. Feise, J. Fricke, M. Matalla, W. John, H. Wenzel, K. Paschke, and G. Erbert, " $12 \mathrm{~W}$ highbrightness single-frequency DBR tapered diode laser," Electron. Lett. 44, 1253-1255 (2008).

3. T. Y. Fan, "Laser beam combining for high-power, high-radiance sources," IEEE J. Sel. Top. Quantum Electron. 11, 567-577 (2005)

4. F. X. D'Amato, E. T. Siebert, and C. Roychoudhuri, "Coherent operation of an array of diode-lasers using a spatial filter in a Talbot cavity,” Appl. Phys. Lett. 55, 816-818 (1989).

5. R. Huang, B. Chann, L. Missaggia, S. Augst, M. Connors G. Turner, A. Sanchez-Rubio, J. Donnelly, J. Hostetler, C. Miester, and F. Dorsch, "Coherent combination of slabcoupled optical waveguide lasers," Proc. SPIE 7230, 72301G (2009).

6. B. Liu, Y. Liu, and Y. Braiman, "Coherent beam combining of high power broad-area laser diode array with a closed-V-shape external Talbot cavity," Opt. Express 18, 7361-7368 (2010).

7. J. Yaeli, W. Streifer, D. R. Scifres, P. S. Cross, R. L. Thornton, and R. D. Burnham, "Array mode selection utilizing an external cavity configuration," Appl. Phys. Lett. 47, 89-91 (1985).

8. C. J. Chang Hasnain, J. Berger, D. R. Scifres, W. Streifer, J. R. Whinnery, and A. Dienes, "High-power with high-efficiency in a narrow single-lobed beam from a diode-laser array in an external cavity," Appl. Phys. Lett. 50, 1465-1467 (1987).

9. B. L. Volodin, S. V. Dolgy, E. D. Melnik, E. Downs, J. Shaw, and V. S. Ban, "Wavelength stabilization and spectrum narrowing of high-power multimode laser diodes and arrays by use of volume Bragg gratings," Opt. Lett. 29, 1891-1893 (2004).

10. S. Yiou, F. Balembois, P. Georges, and J.-P. Huignard, "Improvement of the spatial beam quality of laser sources with an intracavity Bragg grating," Opt. Lett. 28, 242-244 (2003).

11. G. Lucas-Leclin, D. Pabœuf, P. Georges, J. Holm, P. Andersen, B. Sumpf, and G. Erbert, "Wavelength stabilization of extendedcavity tapered lasers with volume Bragg gratings," Appl. Phys. B 91, 493-498 (2008).

12. D. Mehuys, W. Streifer, R. G. Waarts, and D. F. Welch, "Modal analysis of linear Talbot-cavity semiconductor-lasers," Opt. Lett. 16, 823-825 (1991).

13. C. J. Chang-Hasnain, A. Dienes, J. R. Whinnery, W. Streifer, and D. R. Scifres, "Characteristics of the off-centered apertured mirror external cavity laser array,” Appl. Phys. Lett. 54, 484-486 (1989).

14. J. K. Butler, D. E. Ackley, and D. Botez, "Coupled-mode analysis of phase-locked injection-laser arrays," Appl. Phys. Lett. 44, 293-295 (1984) 
15. O. Andrusyak, V. Smirnov, G. Venus, V. Rotar, and L. Glebov, "Spectral combining and coherent coupling of lasers by volume Bragg gratings," IEEE J. Sel. Top. Quantum Electron. 15, 344-353 (2009), and references therein.

16. D. Pabœuf, G. Lucas-Leclin, P. Georges, N. Michel, M. Krakowski, J. J. Lim, S. Sujecki, and E. C. Larkins, "Narrow-line coherently combined tapered laser diodes in a Talbot external cavity with a volume Bragg grating,” Appl. Phys. Lett. 93, 211102 (2008).

17. H. Kogelnik, "Coupled wave theory for thick hologram gratings," Bell Syst. Tech. J. 48, 2909-2947 (1969).

18. J. W. Goodman, Introduction to Fourier Optics (Roberts, 2005).

19. A. F. Glova, N. N. Elkin, A. Y. Lysikov, and A. P. Napartovich, "External Talbot cavity with in-phase mode selection," Quantum Electron. 26, 614-616 (1996).

20. W. Cassarly, J. Ehlert, J. Finlan, K. Flood, R. Waarts, D. Mehuys, D. Nam, and D. Welch, "Intracavity phase correction of an external Talbot cavity laser with the use of liquid crystals," Opt. Lett. 17, 607-609 (1992).

21. M. Krakowski, S. C. Auzanneau, F. Berlie, M. Calligaro, Y. Robert, O. Parillaud, and M. Lecomte, " $1 \mathrm{~W}$ high brightness index guided tapered laser at $980 \mathrm{~nm}$ using $\mathrm{Al}$-free active region materials," Electron. Lett. 39, 1122-1123 (2003).

22. J. J. Lim, T. M. Benson, and E. C. Larkins, "Design of wideemitter single-mode laser diodes," IEEE J. Quantum Electron. 41, 506-516 (2005)

23. J. J. Lim, S. Sujecki, L. Lang, Z. Zhang, D. Pabœuf, G. Pauliat, G. Lucas-Leclin, P. Georges, R. C. MacKenzie, P. Bream, S. Bull,
K. Hasler, B. Sumpf, H. Wenzel, G. Erbert, B. Thestrup P. M. Petersen, N. Michel, M. Krakowski, and E. C. Larkins, "Design and simulation of next-generation high-power, highbrightness laser diodes," IEEE J. Sel. Top. Quantum Electron. 15, 993-1008 (2009).

24. A. Barthelemy, F. Louradour, and V. Couderc, "Wavelengthtunable diffraction-limited operation of a standard high-power diode-laser array using an off-centered extended cavity," Electron. Lett. 28, 2038-2040 (1992).

25. D. Pabøuf, G. Lucas-Leclin, N. Michel, M. Calligaro, M. Krakowski, and P. Georges, "Quasi-diffraction limited emission from an array of tapered laser diodes in volume Bragg grating external cavities," in CLEO/Europe and EQEC 2009 Conference Digest (Optical Society of America, 2009), paper CB12_5.

26. M. Kelemen, J. Weber, G. Kaufel, G. Bihlmann, R. Moritz, M. Mikulla, and G. Weimann, "Tapered diode lasers at $976 \mathrm{~nm}$ with $8 \mathrm{~W}$ nearly diffraction limited output power," Electron. Lett. 41, 1011-1013 (2005)

27. The phase plate has been designed and made by PowerPhotonic, Ltd., http://www.powerphotonic.co.uk.

28. C. J. Corcoran and F. Durville, "Experimental demonstration of a phase-locked laser array using a self-Fourier cavity," Appl. Phys. Lett. 86, 201118 (2005).

29. J. R. Leger, G. J. Swanson, and W. B. Veldkamp, "Coherent laser addition using binary phase gratings,” Appl. Opt. 26, 4391-4399 (1987) 